\title{
The Mental Health Evaluation System of College Students Based on Data Mining
}

\author{
Peng Li iD \\ Faculty of Marxism, Lishui University, LiShui Zhejiang 323000, China \\ Correspondence should be addressed to Peng Li; lsxymkszyxylp@lsu.edu.cn
}

Received 14 December 2021; Revised 7 January 2022; Accepted 12 January 2022; Published 21 February 2022

Academic Editor: Baiyuan Ding

Copyright (C) 2022 Peng Li. This is an open access article distributed under the Creative Commons Attribution License, which permits unrestricted use, distribution, and reproduction in any medium, provided the original work is properly cited.

\begin{abstract}
In order to solve the problems of high misevaluation rate and low work efficiency in the current mental health evaluation process of college students, a mental health evaluation system based on data mining algorithms is proposed. First, analyze the research status of college students' mental health evaluation and data mining algorithms and build a mental health evaluation system framework; then, collect college students' mental health questionnaire data, use the Apriori algorithm based on a three-dimensional matrix to analyze and classify, traverse each attribute of each transaction in the two-dimensional matrix, and directly obtain the frequent item set, frequent binomial set, and frequent three-item set by reading the three-dimensional attribute matrix and the mental health evaluation data to obtain mental health intelligent evaluation results. Finally, specific simulation experiments are used to analyze the feasibility and superiority of the mental health intelligent evaluation system. The results show that the system in the article overcomes the shortcomings of the current mental health intelligent evaluation system, improves the accuracy of mental health intelligent evaluation, improves the efficiency of mental health intelligent evaluation, and the system is more stable, which can meet the actual requirements of current college students' mental health evaluation.
\end{abstract}

\section{Introduction}

The psychological quality education of college students is one of the important contents of higher education. Through the psychological quality education, it is expected to cultivate the good character and will quality of college students, enhance the psychological adaptability and endurance ability, and promote the development of college students' physical and mental health [1-4]. With the effective support of relevant national policies, the psychological quality education of colleges and universities has gradually developed, and the professional team of psychological health quality education in colleges and universities has begun to take shape. Considering the coordination between student health management and career development, the related issues of psychological counseling have attracted more and more attention from school administrators [5-7]. However, in real life, whether facing college groups or other social groups, due to their own obstructive reasons, they are often unwilling to seek help from the counseling center so that they cannot get professional help in time when they have unhealthy manifestations such as psychological depression. Online psychological counseling can avoid this problem well, help people answer mental health problems more widely, provide various online communication and knowledge popularization, and obtain psychological support by browsing other people's comments, thereby effectively improving the efficiency of mental health management consulting, to promote the development of students' mental health and improve their psychological quality [7-11].

Mental health education for college students in China started in the 1990s and initially established the leading role of college students' mental health education courses in educational work, but it still has the problem of poor curricula education effects. Many scholars have conducted research on the issue of mental health consultation [12-14]. Literature [15] pointed out that the psychological problems of college students have become an important factor in integrating into the society. In order to effectively alleviate the psychological pressure of students, it designed a student 
mental health consulting service system based on the Web and designed the system technology according to actual needs, but the safety of the system needs to be improved. Literature [9] designed a multisource mental health questionnaire, visualized the data obtained from the survey, studied the attribute correlation between various types of data, focused on analyzing the uncertain mental health data, and comprehensively judged students. For psychological problems, this method works well, but the data quality is not high. Literature [16] designed a mental health service system for the elderly based on Internet technology and analyzed it in combination with application cases. The practicality is good, but it takes a long time. Literature [17] introduced artificial intelligence, designed a knowledge system with a multilevel framework by building a knowledge base, using Bayesian and certainty factor methods to reason about data, and designed a psychological consultation expert system, but the system is not safe enough. Literature [18], with the support of Android technology, designed a mobile-end student mental health consultation system based on the $\mathrm{C} / \mathrm{S}$ architecture, analyzed the client functional modules in detail, and designed the system architecture by combining the complete communication between the client and the server. The system function is clarified, but it takes a long time. In the 21st century, the country has gradually begun to pay more attention to the mental health of college students. The State Council (2004) No. 16 "Opinions on Further Strengthening and Improving the Ideological and Political Education of College Students" requires major colleges and universities across the country to attach importance to the mental health education of college students. Jiao Sizheng Hall (2011) No. 1 "Basic Construction Standards for Students' Mental Health Education in General Colleges and Universities (Trial)" requires colleges and universities to incorporate college students' mental health education into the school's talent training system and provide psychological counseling services through multiple channels. At the same time, more and more colleges and universities have begun to gradually establish and improve the psychological files of college students, paying attention to the monitoring and prevention of students' psychological crises. At present, many colleges and universities have to conduct a special mental health assessment (SCL-90) for freshmen when they first enter the school, to understand the psychological status of the students, to find out the psychological crisis in the students in time, to promptly guide and intervene according to the psychological status, and to deal with the students. Provide targeted psychological counseling. At the same time, it has gradually begun to consciously save the relevant data on the mental health of college students in a certain way to lay the foundation for the formation of psychological files.

With the development of science and technology, computer applications have gradually entered the operation and management work of all walks of life. Many colleges and universities have adopted psychological evaluation systems to evaluate the mental health of students. Through the psychological evaluation system, college psychological counseling staff can quickly collect students' psychological conditions and make judgments on the results, improve work efficiency, reduce work intensity, and provide great help to a certain extent. At the same time, the database also accumulates a large amount of psychological data [19-22]. However, most colleges and universities currently conduct psychological evaluations based on the access, query, statistics, and backup of basic psychological information of students. They have not conducted in-depth analysis of large amounts of psychological data, and have not fully utilized these data to dig out the hidden information. Some of the information knowledge grasp the trend of students' psychological development, so it cannot effectively provide decision-making assistance to the psychological counseling work [23]. Data mining is to dig out the hidden information knowledge from massive data. The development and application of data mining technology in the retail industry, financial industry, telecommunications industry, and other industries have been fruitful. If data mining technology is applied to the development of mental health management systems, a large number of fuzzy records in student mental health files will be removed through related algorithms. The random data information is processed, and the potential information is unearthed. To a certain extent, it will help the psychological counseling teacher to more scientifically and quickly conduct student psychological judgment and prevention, timely conduct psychological counseling and intervention, improve work efficiency, and reduce psychological incidents which happened [24].

In order to find a more effective psychological consultation system, this paper designs a mental health education consultation management system based on the Apriori algorithm on the basis of existing research results. By introducing data mining technology, it can dig out what is needed in massive data. On the basis of ensuring the quality of psychological quality teaching, improve the effect of psychological quality education for college students.

\section{The Overall Structure of the Mental Health Evaluation System for College Students}

In order to enable people to have an accurate understanding of their own mental health and, at the same time, to promote the scientific and informatization of mental health guidance, this paper constructs an intelligent evaluation system for mental health based on data mining, using scientific mental health evaluation tools. To comprehensively reflect the user's mental health level, the overall structure of the system is shown in Figure 1.

The mental health intelligent evaluation system based on data mining mainly includes four modules: basic information management module, evaluation test question management module, evaluation result analysis module, and personal psychological evaluation module.

(1) The basic information management module is the basic function in the text system, and its main function is to manage the basic information in the text system. This module has the function of personal information synchronization and evaluation type management. The former can synchronize the 


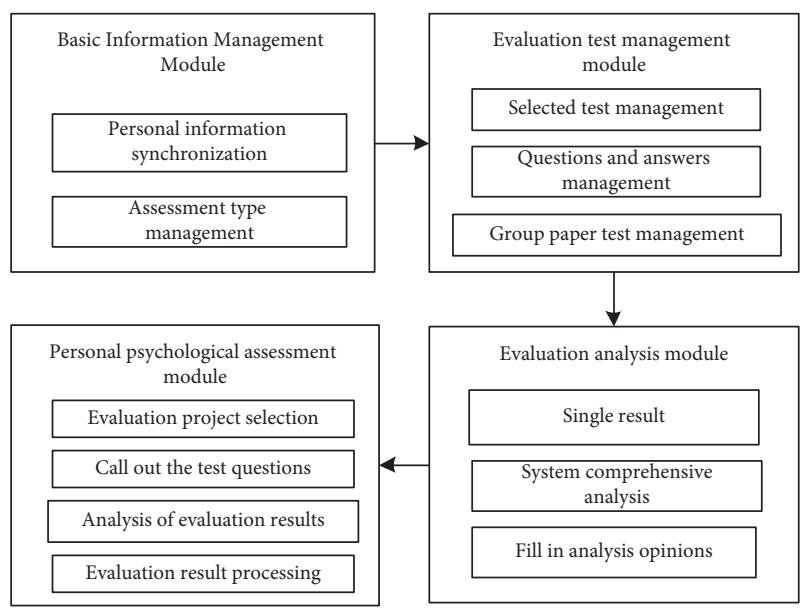

FIgURE 1: Overall diagram of the system structure.

personal information filled in by the user, accurately track the user's real-time personal situation, and ensure the accuracy of the follow-up evaluation of this system. The latter can manage the system in this paper. The latter can manage the assessment types in the system in a unified manner, and add or delete assessment types according to user needs. In the process of using the system, users have many types of choices, high versatility, and powerful overall system functions and can be widely used in the crowd.

(2) The evaluation test question management module is an important part of this system. The quality of the evaluation test questions seriously affects the correctness of the evaluation results. This module mainly includes three types of test questions: choice test questions, question answering test questions, and test paper grouping test questions. The form of multiple choice questions can be single-choice or multiple choice questions. There is a strong correlation between the test questions. Answers are made in the evaluation test questions through the selection method, and the answers are selected according to the user's choice. With different jumps to different question types to complete the answer, the answer points of each question are different. The question form of the essay questions is a question and answer, which is designed according to different types of assessment. The test question format of the test paper combines the choice test and the essay test. The above two test question types are combined in different evaluation types, and the jump to the multiple choice or essay is based on the user's answer, and the jump to the essay is terminated after the jump to the essay. This system can effectively reduce the user's psychological precautions through the skipping of thinking in the choice of test questions, increase their interest in testing, make the test easier to be accepted by them, and obtain a deeper understanding of the user's psychological state through the question and answer test.
(3) The evaluation result analysis module is responsible for statistically analyzing the psychological evaluation results of users, discovering the psychological problems of users in the first time, grasping their psychological conditions, and providing help to users. This module can provide users with the analysis results of a single evaluation, users with the comprehensive analysis results of multiple evaluations, and provides users with the function of filling in analysis opinions, so as to effectively and timely obtain user feedback information on the evaluation results.

(4) The personal psychological evaluation module is the main module in the system of this paper, which highlights the significance of the system of this paper to serve users. The evaluation item selection part provides users with all evaluation items of this system. Users can select evaluation items according to their own needs. The evaluation test question recall function can call out the corresponding evaluation test questions to the user for evaluation according to the evaluation item selected by the user and automatically save the user's evaluation. Answer the result, give the corresponding score, and finally process the user's evaluation result through the evaluation result processing function and feedback the processing result.

\section{Design of Mental Health Evaluation System Based on Data Mining}

The client software of the system in this paper uses the method of psychological evaluation data mining to realize the analysis of evaluation results, processes the obtained user evaluation result data and establishes a database, analyzes the evaluation results through the decision tree algorithm, and obtains an evaluation. Psychological assessment data mining includes extracting data from the database, cleaning the data, selecting the mining mode, and outputting the results. The complete data mining process is shown in Figure 2.

In this paper, an initial dataset is established by using the answering results of the college student survey questionnaire. After preprocessing of data integration, data extraction, data cleaning, and data conversion, a mental health assessment dataset to be mined is obtained, and the Apriori algorithm is used to mine the data. Association rules: Apriori algorithm completes data mining through continuous scanning, candidate item set, and minimum support comparison. Apriori algorithm needs to face a large amount of data when running, which is not conducive to efficient mining. For this reason, the system designed first divides the user psychological counseling database, divides it into multiple equivalent parts, and performs mining operations one by one. Finally, the data mining is completed in a unified manner, and the classification of the data is obtained through the mining results,so as to obtain the specific classification of 


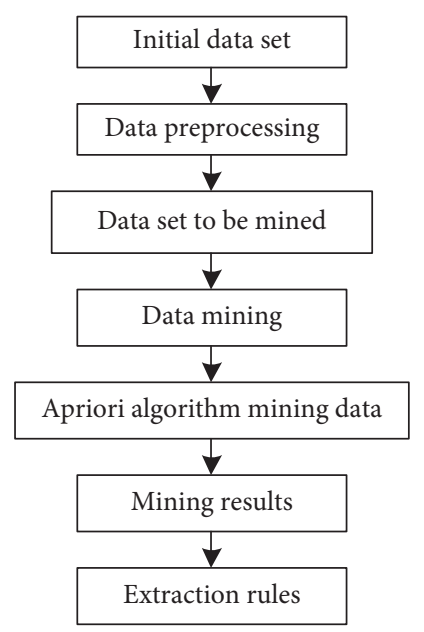

Figure 2: Data mining flowchart of mental health assessment.

the mental health status of college students. The operation steps are as follows:

(1) Divide the university student mental health questionnaire database into $n$ equivalent parts.

(2) Perform frequent item set mining operations for a certain part, obtain data candidate item sets through scanning, compare it with the given minimum support to obtain frequent item sets, and then calculate new items based on frequent item sets and support set of candidates. In the same way, the frequent item set is obtained by comparing the candidate item set with the minimum support, and the operation is repeated continuously according to this step to obtain the final frequent item set.

(3) Use the previous step to process each part of the college student mental health questionnaire database to generate corresponding frequent item sets.

(4) Summarize the frequent item sets of each part and then generate the global frequent item sets. Based on the above, to complete the server-side information processing, we provide an important foundation for the efficient and safe operation of the system.

3.1. Algorithm Description. The Apriori algorithm is used to mine the association rules between the data and find the datasets that often appear in the data values, that is, the frequent item sets; by finding the connections in these sets, it is helpful for decision-making.

Definition 1. The support in association rules refers to the ratio of the number of transactions that contain both $X$ and $\mathrm{Y}$ to all transactions in database $D$, provided that $X=>Y$ is established in database $D$ :

$$
\operatorname{Support}(X \Rightarrow Y)=P(X \cup Y) \text {. }
$$

Definition 2. The confidence in the association rule refers to the ratio of the number of transactions that contain both $X$ and $Y$ to the number of transactions that only contain $X$ in database $D$, namely,

$$
\text { Confidence }(X \Rightarrow Y)=\frac{\operatorname{Support}(X \Rightarrow Y)}{\operatorname{Support}(X)}=P(Y / X) \text {. }
$$

The two attributes of confidence and support in association rules are obtained through experience. If they are satisfied,

$$
\operatorname{Sup}(X \Rightarrow Y) \geq \min \_ \text {sup\&conf }(X \Rightarrow Y) \geq \min \_c o n f .
$$

Then, $X$ and $Y$ are strong association rules and vice versa are weak association rules.

3.2. Apriori Algorithm Process Based on Three-Dimensional Matrix. The traditional Apriori algorithm will generate a large number of candidate item sets when the amount of data is large and the analysis categories are many, especially when generating binomial sets and tri-items' sets. And every time a higher level of frequent item sets are generated, the database needs to be rescanned, which will generate a lot of computational redundancy and low efficiency. On the basis of the Apriori algorithm, improvements are made to address these shortcomings of the traditional algorithm. The improved algorithm idea is as follows.

(1) Scan the database first and abstract the database into a two-dimensional matrix based on the attributes contained in all its transactions, which are used to store all the information in the database.

(2) Traverse each attribute of each transaction in the two-dimensional matrix. By reading two different attributes in the same transaction each time without repeating the reading, the three-dimensional upper triangular attribute matrix Matrix $(i, j, k)$ is established, and the coordinates are established according to the corresponding attributes. The coordinate intervals in the three dimensions are all $[1, \mathrm{~N}](N$ is the largest attribute type). During the scanning process, each time the coordinates are repeated, the corresponding weight is increased by one, and the matrix can be expressed as

$$
\operatorname{Matrix}(i, j, k)=\operatorname{Matrix}(i, j, k)+1 \text {. }
$$

(3) Secondly, by reading the three-dimensional attribute matrix, we can directly obtain the frequent item set, frequent binomial set, and frequent three-item set. The space diagonal of the first hexagram limit of the three-dimensional matrix is the support of frequent item sets, and the coordinates $(i, j, j)$ on the corresponding plane are the support of frequent binomial sets and coordinates $(i, j, k)$. It is the support degree of the corresponding three-item set.

(4) In the transaction with the number of attributes less than $k$, there must be no possibility of containing $k$ itemsets. Therefore, after getting the frequent threeitem set, scan the database. Delete the affairs that 
contain no more than four attributes, and simplify the database.

(5) Use the standard Apriori algorithm for subsequent calculations through the frequent three-item sets that have been obtained. The specific algorithm flow is shown in Figure 3.

\section{Experiment Analysis}

Randomly select 1,000 college students from a certain university as the experimental subjects, and compare the mental states of employees measured by this system and professional psychologists. 1 represents neuroticism, 2 represents compulsive paranoia, 3 represents emotional instability, 4 represents emotional weakness, and 5 represents communication sensitivity. The result is shown in Figure 4.

According to Figure 4, it can be seen that the psychological evaluation results of the system in this paper are basically consistent with those of professional psychologists, which proves that not only the system in this paper is effective but also its evaluation results are also very good. At the same time, it can be seen that the number of employees with neurasthenia accounted for the largest proportion. The number of employees with forced paranoia and emotional instability were $27 \%$ and $22 \%$, respectively. The number of employees with neuroticism and communication sensitivity was relatively small. The reason was the high work pressure and competition of the unit. The pressure is strong. These employees can effectively reduce the proportion of various psychological problems after a period of emotional resolution based on the results of the processing opinions given by the system of this paper, which proves that the system of this paper is practical and can provide users with effective solutions to psychological problems.

In order to verify the data processing efficiency of the system in this paper, the Internet-based mental health service system for the elderly and the online forum user mental health automatic evaluation system based on multifeature fusion are used as the comparison system. The time result is shown in Figure 5.

Analyzing Figure 5, we can see that the data processing time of the system in this paper has been lower than that of the other two systems. As the amount of data continues to increase, the data processing time of the three systems has changed. The data processing time of the comparison system 1 and the comparison system 2 has increased sharply with the increase in the amount of information, and the fluctuation range is large and the stability is poor. The increase in processing time is small, the curve is smoother, and the stability is good. After the data volume reaches $5 \times 103 \mathrm{~GB}$, the data processing time gradually stabilizes, which proves that the system in this paper has high data processing efficiency and strong stability.

Different noises were added to the three systems to evaluate the mental state of employees, and the accuracy of the three systems' mental health evaluation was tested. The results are shown in Table 1.

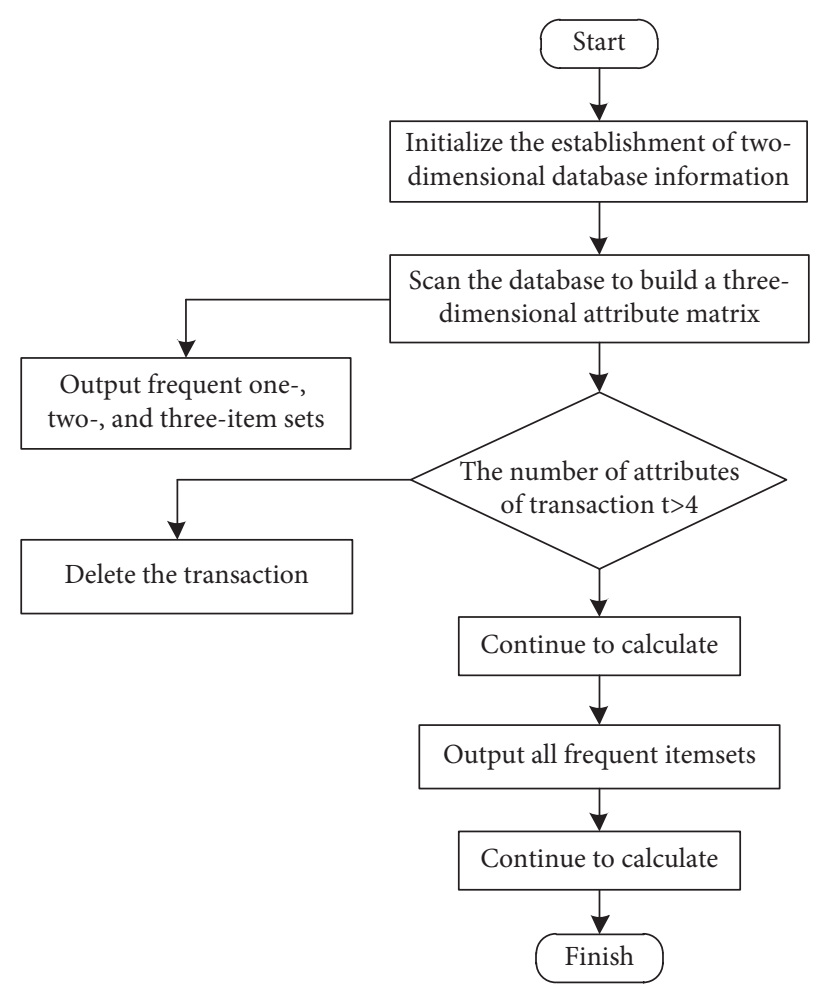

Figure 3: Apriori algorithm flow.

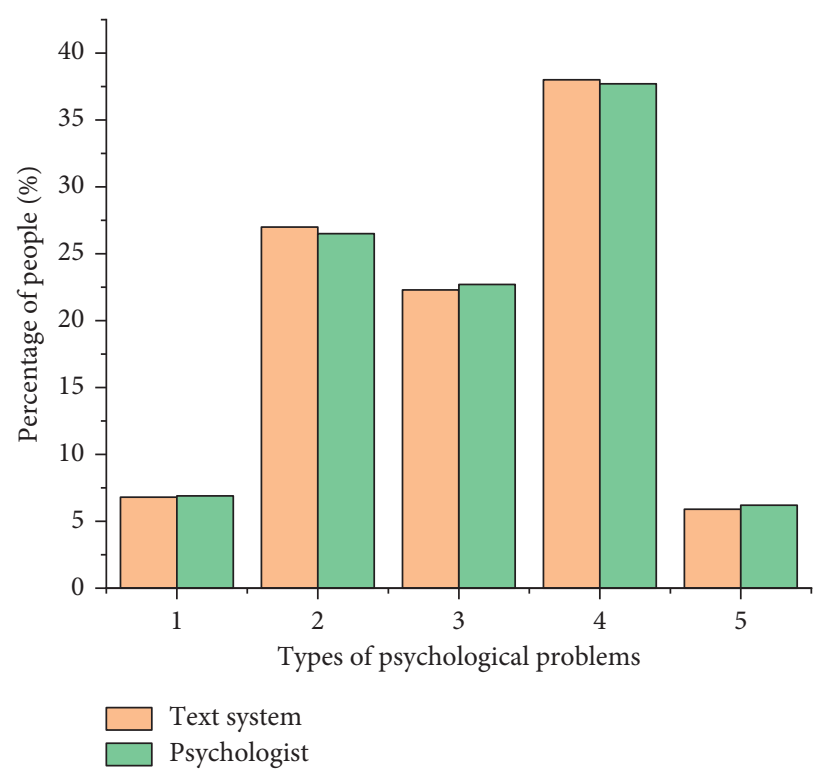

FIgURE 4: Comparison results.

It can be seen from Table 1 that with the continuous increase of noise, the mental health evaluation accuracy of the three systems has decreased, but the mental health evaluation accuracy of the system in this paper has been above $90 \%$, and the accuracy rate has not decreased much. It is relatively stable, while the mental health evaluation accuracy of the other two systems is less than $88 \%$, indicating that the different noises in the three systems have the least impact on the mental health evaluation accuracy of the 


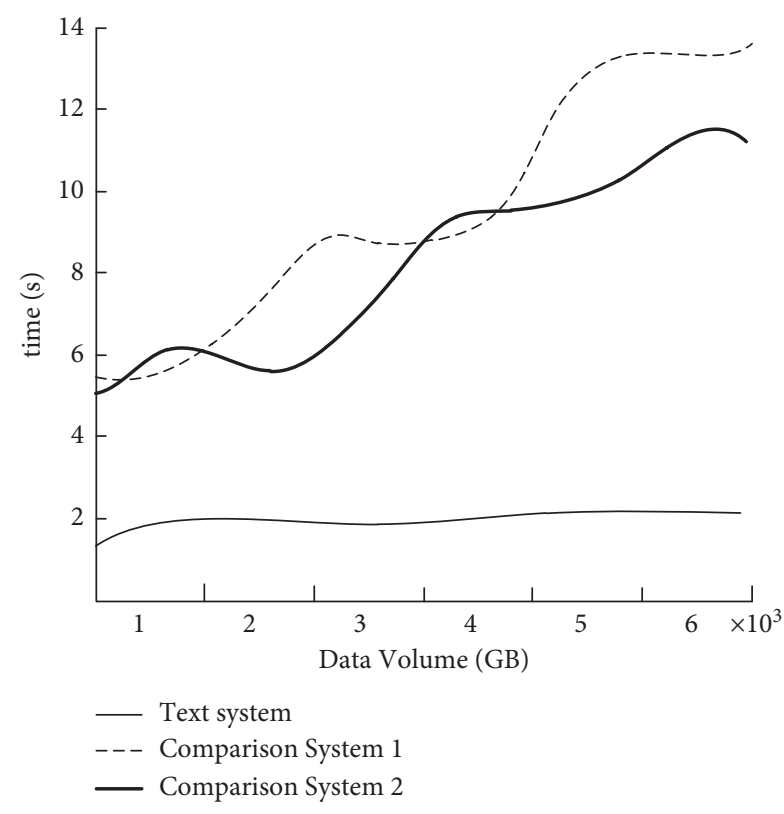

Figure 5: Data processing time of the three systems.

TABle 1: Mental health evaluation accuracy.

\begin{tabular}{lcccc}
\hline Noise $(\mathrm{dB})$ & $20(\%)$ & $40(\%)$ & $60(\%)$ & $80(\%)$ \\
\hline Text system & 93.33 & 92.08 & 92.77 & 91.11 \\
Comparison system 1 & 88.12 & 87.64 & 87.63 & 85.88 \\
Comparison system 2 & 81.68 & 81.62 & 81.11 & 80.33 \\
\hline
\end{tabular}

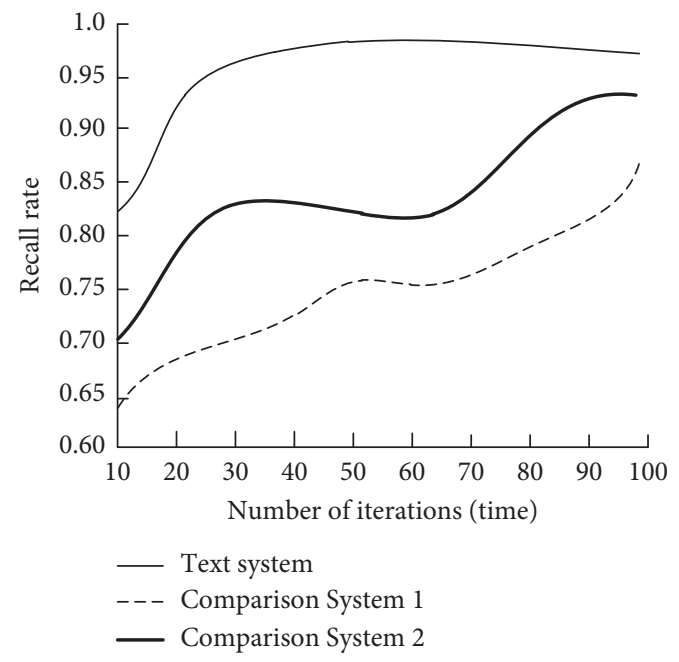

Figure 6: Comparison of system recall rates.

system in this paper. The antinoise performance of the system in this paper is better. The accuracy is high.

The information recall rate can be used to verify the system's data scheduling capability. The higher the recall rate, the more stable it is, and the better the system's data scheduling capability. Test the information recall rate of the three systems in the psychological evaluation, and the results are shown in Figure 6.
It can be seen from Figure 6 that the recall rates of the three systems all increase with the increasing number of iterations. The recall rate of the system in this paper is the highest, and it is stable after the number of iterations reaches 20. It has good recall and adaptive scheduling performance. The recall rate of comparison system 1 and comparison system 2 fluctuates greatly, and the recall rate is lower than that of the system in this paper.

\section{Conclusion}

In order to improve the effect of college students' mental health evaluation, this paper constructs a mental health intelligent evaluation system based on the Apriori algorithm, which can fully help college students understand their own mental health, solve their psychological problems, and enhance their self-awareness. By collecting college students' mental health questionnaire data and using the Apriori algorithm based on the three-dimensional matrix, we analyze and classify the mental health evaluation data and obtain the mental health intelligent evaluation results; finally, the feasibility and superiority of the mental health intelligence evaluation system are analyzed by specific simulation experiments. The results show that the system in the study overcomes the shortcomings of the current mental health intelligent evaluation system, improves the accuracy of mental health intelligent evaluation, improves the efficiency of mental health intelligent evaluation, and has better system stability, which can meet the actual requirements of current college students' mental health evaluation. In order to make the system of this paper more functional and have better development, it is also necessary to improve the analysis of system evaluation results, improve the situation of system data loss during power failure, increase the system's data backup and recording functions, solve the problem of waste of storage space, and make the function of this text system more perfect.

\section{Data Availability}

The dataset used to support the findings of the study can be obtained from the corresponding author upon request.

\section{Conflicts of Interest}

The authors declare that they have no conflicts of interest.

\section{References}

[1] X. Li, Y. Fei, and Y. Fan, "A review of research on occupational mental health of construction workers," China Safety Science Journal, vol. 30, no. 9, pp. 202-210, 2020.

[2] M. Wang, F. Wang, and J. Wang, "Reliability and validity analysis of the Chinese version of the psychological flexibility comprehensive questionnaire in financial institution staff," Chinese Journal of Behavioral Medicine and Brain Science, vol. 27, no. 1, pp. 72-77, 2018.

[3] S. Li and Q. Huang, "A review of researches on the intervention efficacy of gardening activities based on quantitative measurement of elderly physical and mental health 
indicators," Journal of Northwest University, vol. 50, no. 6, pp. 852-866, 2020.

[4] M. Wang, F. Han, and L. Jia, "Meta-analysis of the detection rate of depressive symptoms and related factors in college students," Chinese Mental Health Journal, vol. 34, no. 12, pp. 1041-1047, 2020.

[5] L. Wen, N. Zhang, and Z. Yu, "Meta-analysis of the relationship between emotion regulation and mental health of children and adolescents," Chinese Journal of Clinical Psychology, vol. 28, no. 5, pp. 1002-1008, 2020.

[6] S. Zhang, T. Liu, and F. Xia, "Social network analysis of interpersonal relationship and mental health of college students," Chinese Mental Health Journal, vol. 34, no. 10, pp. 855-859, 2020.

[7] J. Liang and H. Luo, "The application of big data mining methods in the psychological early warning system of college students," Chinese School Health, vol. 39, no. 12, pp. 18211824, 2018.

[8] D. Liu, X. Xia, and C. Wan, "Automatic evaluation of online forum users' mental health based on multi-feature fusion," Chinese Journal of Computers, vol. 42, no. 7, pp. 1553-1569, 2019.

[9] X. Chen, M. Tong, and S. Chen, "Visual analysis of multisource mental health questionnaire data for college students," Journal of Computer Aided Design and Graphics, vol. 32, no. 2, pp. 181-193, 2020.

[10] Z. Liu and X. Song, "A multivariate decision tree algorithm that can be used for categorized attribute data," Journal of Northeastern University, vol. 41, no. 11, pp. 1521-1527, 2020.

[11] J. Liang and H. Luo, "The application of big data mining methods in the psychological early warning system of college students," Chinese School Health, vol. 39, no. 12, pp. 18211824, 2018.

[12] H. Gong, "Construction of a mental health management platform for college students based on new media technology," Library Work and Research, vol. 1, no. 10, pp. 124-128, 2017.

[13] K. Jia, H. Li, and Y. Yuan, "Application of data mining based on Apriori algorithm in mobile medical system," Journal of Beijing University of Technology, vol. 43, no. 3, pp. 394-401, 2017.

[14] F. Zhao and B. Liu, "Association analysis of college students' performance based on improved Apriori algorithm," Journal of Qiqihar University (Natural Science Edition), vol. 34, no. 1, pp. 11-15, 2018.

[15] H. Wang, "Design and implementation of a web-based mental health consultation service system for college students," Journal of Xi'an University of Arts and Science (Natural Science Edition), vol. 20, no. 1, pp. 72-76, 2017.

[16] Y. Qian, D. Chen, and M. Zhu, "Design of mental health service system for the elderly based on Internet technology," Packaging Engineering, vol. 38, no. 22, pp. 53-59, 2017.

[17] L. Yang, "Psychological consultation expert system reasoning model design," Computer and Digital Engineering, vol. 46, no. 6, pp. 1145-1148, 2018.

[18] Y. Yin, Design and Implementation of a mobile College Student Mental Health Consulting Service System Based on C/S Architecture [D], Jilin University, Changchun, 2018.

[19] L. Deng, J. Liang, and B. Li, "The status quo of mental health education in primary and secondary schools: the different perspectives of psychological teachers and school administrators," Teacher Education Research, vol. 30, no. 4, pp. 58-64, 2018.
[20] R. Su, The Design and Implementation of an Android-Based Mental Health Management System for College Students, Shandong University, Jinan, 2017.

[21] Y. Zhang, C. Hu, and S. Huang, "Data mining and analysis method of secondary equipment defects based on Apriori algorithm," Automation of Electric Power Systems, vol. 41, no. 19, pp. 147-151, 2017.

[22] Z. Xie and P. Wang, "Parallel matrix Apriori algorithm based on MapReduce architecture," Computer Application Research, vol. 34, no. 2, pp. 401-404, 2017.

[23] R. Zhong and H. Wang, "Specific data query technology in the university cloud computing management system based on data mining," Modern Electronic Technology, vol. 41, no. 2, pp. 130-132, 2018.

[24] M. Luo, "Research on students' mental health based on data mining algorithms," J Healthc Eng, vol. 20211382559 pages, 2021, PMID: 34733450; PMCID: PMC8560244, Article ID 1382559. 